\title{
Electroacupuncture Treatment Improves Learning-Memory Ability and Brain Glucose Metabolism in a Mouse Model of Alzheimer's Disease: Using Morris Water Maze and Micro-PET
}

\author{
Jing Jiang, ${ }^{1}$ Kai Gao, ${ }^{2}$ Yuan Zhou, ${ }^{1}$ Anping Xu, ${ }^{1}$ Suhua Shi, ${ }^{1}$ Gang Liu, ${ }^{3}$ and Zhigang Li ${ }^{1}$ \\ ${ }^{1}$ Beijing University of Traditional Chinese Medicine, Beijing 100029, China \\ ${ }^{2}$ Institute of Laboratory Animal Science, Chinese Academy of Medical Sciences \& Comparative Medical Center, \\ Peking Union Medical College, Beijing 100021, China \\ ${ }^{3}$ Community Health Service Center of Dongcheng District, Beijing 100010, China
}

Correspondence should be addressed to Zhigang Li; lizhigang620@126.com

Received 3 December 2014; Revised 12 January 2015; Accepted 14 January 2015

Academic Editor: Mani Vasudevan

Copyright (C) 2015 Jing Jiang et al. This is an open access article distributed under the Creative Commons Attribution License, which permits unrestricted use, distribution, and reproduction in any medium, provided the original work is properly cited.

\begin{abstract}
Introduction. Alzheimer's disease (AD) causes progressive hippocampus dysfunctions leading to the impairment of learning and memory ability and low level of uptake rate of glucose in hippocampus. What is more, there is no effective treatment for AD. In this study, we evaluated the beneficial and protective effects of electroacupuncture in senescence-accelerated mouse prone 8 (SAMP8). Method. In the electroacupuncture paradigm, electroacupuncture treatment was performed once a day for 15 days on 7.5-month-old SAMP8 male mice. In the normal control paradigm and AD control group, 7.5-month-old SAMR1 male mice and SAMP8 male mice were grabbed and bandaged while electroacupuncture group therapy, in order to ensure the same treatment conditions, once a day, 15 days. Results. From the Morris water maze (MWM) test, we found that the treatment of electroacupuncture can improve the spatial learning and memory ability of SAMP8 mouse, and from the micro-PET test, we proved that after the electroacupuncture treatment the level of uptake rate of glucose in hippocampus was higher than normal control group. Conclusion. These results suggest that the treatment of electroacupuncture may provide a viable treatment option for $\mathrm{AD}$.
\end{abstract}

\section{Introduction}

Alzheimer's disease $(\mathrm{AD})$ is a progressive neurodegenerative disease, which is the most widespread cause of dementia and its incidence will continue to increase rapidly as the population ages [1]. It is characterized by the progressive decline of memory and cognitive function and changes in behavior and personality [2]. Despite the fact that extensive research is focused on $\mathrm{AD}$, there is no effective treatment for this disease [3]. Therefore, the therapeutic of AD is urgent to be proposed.

Electroacupuncture (EA) treatment is a type of needling therapy from Journal of Acupuncture and Moxibustion, number 1,1934, which combines needling with electric stimulation, connecting needles of the point group concerned (2 points make up a group) with pulse current from the electric stimulator [4]. There is a dual-directional pulse current (intermittent oscillatory current), sin wave, square wave, and so forth, with characteristics such as continuous wave, sparse-dense wave, intermittent wave, undulate wave, and saw tooth wave. The frequency most commonly used is 11000 times/sec. over 1000 times/sec. which is used less. What is more, the proper intensity of the stimulation is marked by a muscular twitch around the acupoint and a comfortable sensation [5]. EA treatment has advantages like many kinds of oscillation waves, wide range of frequency, and stable function [6].

So far, EA treatment has yielded neuroprotective function in animal models of depression, spinal cord injury $[7,8]$, cerebral ischemia-reperfusion injury [9-12], stork [9, 10, 12, 13], and many kinds of pain [14-20]. Clinically, EA treatment has been shown to have efficacy in curing many kinds of neurological disease, such as depression [21, 22], spinal cord injury [23-25], cerebral ischemia-reperfusion injury [26], stork [27-29], and many kinds of pain [30-36]. Thus, some of researcher proposed that since the EA treatment could 
protect the central nervous system it may be used as an alternative treatment for $\mathrm{AD}$ [37]. However, more research is needed to prove this conclusion.

The aim of this study was to assess the efficacy of EA treatment in curing $\mathrm{AD}$. We utilized the mouse model of $\mathrm{AD}$, senescence-accelerated mouse prone 8 (SAMP8), which develops the learning and memory impairment and the mood disorder. Here, we present the ethology and in vivo imaging evidence that EA treatment over a period of half a month improves the learning and memory ability and brain glucose metabolism in $\mathrm{AD}$, specifically in the hippocampus of the SAMP8 mouse. This finding extends our previous EA work in the models of the central nervous injury to demonstrate that EA treatment is also effective in protecting the brain against chronic insults due to $\mathrm{AD}$-related disease.

\section{Method and Materials}

2.1. Animals. Senescence-accelerated mouse prone 8 (SAMP8) and the cognate normal senescence-accelerated mouse-R1 (SAMR1) breeding pairs were kindly provided by Professor Takeda at Kyoto University, Japan [38]. The animals were housed in a barrier facility of the Experimental Animal Centre of First Teaching Hospital of Beijing University of Traditional Chinese Medicine and under live conditions of controlled temperature $\left(24 \pm 2^{\circ} \mathrm{C}\right)$, a $12 \mathrm{~h} / 12 \mathrm{~h}$ dark/light cycle, and sterile drinking water and standard pellet diet ad libitum. All experiments were performed according to the National Institute of Health Guide for the Care and Use of Laboratory Animals (NIH publications number 80-23). Thirty 7.5-month-old SAMP8 male mice were divided into two groups ( $n=10$ per group): SAMR1 normal control (Rc) group, SAMP8 Alzheimer's disease control (ADc) group, and SAMP8 electroacupuncture (EA) group.

2.2. Acupuncture Manipulation. In the EA group, electroacupuncture treatment was performed once a day for 15 days (no treatment on the eight day). The prescription of acupuncture points included DU20 Baihui, DU 26 Shuigou, and EX-HN3 Yintang (the significant extra point). The locations of these points were according to the National Acupuncture Society for Experimental Research developed the "laboratory animal acupuncture atlas". Huatuo card 30\#, 0.5 inch needle was used for treatment. Pricking method was used for DU 26 Shuigou; flat thorn method was used for DU20 Baihui and EX-HN3 Yintang. Needle depth was $0.5 \mathrm{~cm}$ and taped. The needle handle was connected with HANSLH202 electroacupuncture device (Peking University Institute of Science Nerve and Beijing Hua Wei Industrial Development Company), sparse wave, $2 \mathrm{~Hz}$ of the frequency, $2 \mathrm{~V}$ of the voltage, and $0.6 \mathrm{~mA}$ of the current intensity.

In the Rc group and $\mathrm{ADc}$ group, do not do any treatment under the same rearing conditions, while grabbing and bondage the mice in order to ensure the same treatment conditions, once a day, 15 days.

2.3. Morris Water Maze Behavioral Test. The Morris water maze consisted of a circular tank $(90 \mathrm{~cm}$ in diameter, $50 \mathrm{~cm}$ in height) filled with water to a depth of $29 \mathrm{~cm}$ maintained at 24 $\pm 1^{\circ} \mathrm{C}$ and rendered opaque with blue-black ink. A removable circular platform ( $9.5 \mathrm{~cm}$ diameter, $28 \mathrm{~cm}$ height) with its top surface $1 \mathrm{~cm}$ below the water was located inside the pool. The area of the pool was conceptually divided into four quadrants (NE, NW, SW, and SE) of equal size. Data were collected by a video camera (TOTA-450Ш, Japan) which was fixed to the ceiling of the room and connected to a video recorder and an automated tracking system (China Daheng Group, Beijing, China).

In this behavioral test, mice are placed in the pool of water containing a platform just below the surface of the water. They escape from the maze when they find the platform. Distal visual cues are arrayed around the room, and in general, mice are able to learn the location of the hidden platform based on these cues.

2.4. Hidden Platform (Place) Testing. This portion of the test assesses the ability of the mice to find the platform under conditions where they cannot directly see it but must either remember it is relative to external cues or perform a search for it. The platform was placed $1 \mathrm{~cm}$ under the surface of the water, and the water was opaque by a suspension of dark blue, nontoxic tempera paint. The platform was placed in a different location from that used in the visible platform testing. Each mouse was released from one of 4 locations and had $60 \mathrm{~s}$ to search for the hidden platform. At the end of each trail, the mouse was placed on the platform or allowed to stay on the platform for $15 \mathrm{~s}$. Prominent spatial cues were arrayed around the room. The investigator is also a powerful spatial cue and always sat in the same location during each trail after releasing the mouse. Eight trails per day for 4 consecutive days were performed with the location of the platform kept constant. We recorded the time that the mouse found the platform needed, and we call it escape latency.

2.5. Probe Trail. The day after the completion of hidden platform testing, the platform was removed, and each mouse was placed in the pool once for $60 \mathrm{~s}$, starting from the same starting location as was used first in hidden platform testing. The time spent swimming in the quadrant where the platform had been was recorded. This is considered to be the most specific test for spatial memory. We recorded the time that the mouse spent in the platform quadrant and calculated the percentage of total time spent in swimming to the platform quadrant.

2.6. Micropositron Emission Tomography. Before experiments, each mouse (7.5 months, 28 32 g) for blood glucose monitoring, the results showed the normal range (7.0 $10.1 \mathrm{mmol} / \mathrm{L})$ could be used for micro-PET detective $\left({ }^{18} \mathrm{~F}\right.$ FDG PET tracer was provided by the Chinese Medicine Research Institute PET Room; PET imaging system using Siemens INVEON PET/CT imaging system). Six hours of water deprivation before the experiment. The mice were placed in the suction chamber, inhaling the oxygen mixed with $1.5 \%$ isoflurane to be anesthetized. After complete anesthesia, approximately $14.8 \sim 16.5 \mathrm{MBq}{ }^{18} \mathrm{~F}$-FDG PET tracers 
were injected via vena of tail. After the ${ }^{18}$ F-FDG PET tracer uptake for $60 \mathrm{~min}$, the mice were placed on the scan bed in prone position, the mice and scanner long axis were parallel, and the head of mouse was located within the scanner field of view. Then the micropositron emission tomography began to collect the image. During this progress, the mice were anesthetized by the oxygen mixed with $1.5 \%$ isoflurane (1 L/min).

2.7. Micropositron Emission Tomography Image Reconstruction. Filtered back projection (FBP) and CT photon attenuation correction were used for image reconstruction. Dynamic micro-PET image frames are taken $30 \mathrm{~s} /$ frames.

2.8. Region of Interest Selection. The three-dimensional region of interest technology was applied for manual selection of the hippocampus three-dimensional region of interest in transverse, coronal, and sagittal planes. Then calculate the uptake rate of per gram with the region of interest.

2.9. Statistical Method. All data were analyzed by SPSS (version 17.0; SPSS, Inc., Chicago, IL, USA). All measurements were performed by an independent investigator blinded to the experimental conditions. The results in the figures are expressed as the mean \pm standard deviation. Differences within or between normal distributed data were analyzed by analysis of variance (ANOVA) followed by Huynh-Feldt test (for Morris water maze test). Statistical significance was set at $P<0.05$.

\section{Results}

3.1. Effect of Electroacupuncture in Spatial Learning Ability of SAMP8 Mouse in the Morris Water Maze Test. The effect of electroacupuncture in spatial location ability of SAMP8 mouse in the WMW test is elucidated in Figure 1(a). We can see that with the training time extension, the escape latency of all groups had shown a downward trend (Figure 1(b)). The $\mathrm{AD}$ control group showed marked retardation in the escape latency, probably due to the memory deficits resulting from the rapid aging process impairment of learning and memory. The analysis of the escape latency revealed that the mouse in EA group had significantly reduced the escape latency compared with the AD control group $(P<0.05$, Figure $1(\mathrm{c}))$.

3.2. Effect of Electroacupuncture in Spatial Memory Ability of SAMP8 Mouse in the Morris Water Maze Test. To investigate the effect on spatial memory ability, the performance in the probe trial on day 6 was examined by analyzing the percentages of time spent swimming to the expected position of the platform. A higher percentage of time spent in the platform quadrant is interpreted as a higher level of memory retention [39]. In this probe trial, we found that compared with $\mathrm{AD}$ control group, EA group spent higher time in the platform quadrant $(P<0.01)$. What is more, in percentage of time spent in the platform quadrant, EA group and normal group had no significant difference in statistics $(P=0.223$, Figure 2).
3.3. PET Imaging of Mice Hippocampus. Because of the effects of the tail vein injection, condition of anesthesia, and the metabolism of the ${ }^{18} \mathrm{~F}-\mathrm{FDG}$, four animals of each group could successfully guarantee the completion of micro-PET test.

Use the same color standard and color code from top high to the bottom low to display the metabolic rate of the glucose. The left of the observer is the right of the animal. From the image, after the treatment of electroacupuncture the ${ }^{18} \mathrm{~F}$-FDG of hippocampus is higher than Alzheimer's disease group (Figure 3).

3.4. ${ }^{18}$ F-FDG Uptake Rate of per Gram in Hippocampus Tissue. To study that the treatment of electroacupuncture corresponds to enhancing the glucose metabolic activity in hippocampus, ${ }^{18} \mathrm{~F}$-FDG PET scan was performed on the mice. The result showed that after treatment of electroacupuncture the uptake rate of ${ }^{18} \mathrm{~F}-\mathrm{FDG}$ in hippocampus was higher than Alzheimer's disease group and normal control group (Figure 4).

\section{Discussion}

In the current research, we studied the effect of electroacupuncture on animal model of Alzheimer's disease using Morris water maze and micro-PET and aimed to find that whether the treatment of electroacupuncture can improve the condition of Alzheimer's disease. Using the Morris water maze, we found after the treatment of electroacupuncture that the spatial learning and memory ability of the SAMP8 mouse had improved. Further, the result of micro-PET revealed that treatment of electroacupuncture can increase the uptake rate of glucose in hippocampus of SAMP8 mouse. These findings from animal behavior and in vivo imaging lead us to conclude that the treatment of electroacupuncture may play a curable role in Alzheimer's disease, particularly in the learning and memory ability.

\subsection{Alzheimer's Disease in Traditional Chinese Medicine and} the Acupuncture Prescription. Alzheimer's disease belonged to encephalopathy in Chinese medicine. It is caused by deficiency of jing and blood, with the aged condition, leading to serious brain function disorder. It is characterized by forgetful and personality changes [40]. In the treating principle of Chinese medicine, according to the principle "the brain is the house of mentality," "the Governor Vessel ... entering the brain and extending up to the very top of the head," based on the close relationship brain-mentality and brainGovernor Vessel, we proposed "dredging the Governor Vessel and awakening mind" method to treat Alzheimer's disease.

In the selection of acupoints, we chose DU20 Baihui, DU 26 Shuigou, and EX-HN3 Yintang as the main points. DU20 Baihui, a meridian point of the Governor Vessel, and the meeting point of Governor Vessel, the three Yang Meridians of the hand and foot, from the A-B Classic of Acupuncture and Moxibustion (zhen jiu jia yi jing), also named Sanyangwuhui, Dianshang, Wuhui. It is located on the head, 5 cun directly above the midpoint of the anterior hairline. Its indication is headache, dizziness, palpitation due to fright, 


\begin{tabular}{|c|c|c|c|c|c|}
\hline Group & Day 1 & Day 2 & Day 3 & Day 4 & Day 5 \\
\hline Normal control group & $41.85 \pm 6.32$ & $32.88 \pm 8.78$ & $27.98 \pm 10.92$ & $24.82 \pm 6.64$ & $19.21 \pm 12.63$ \\
\hline $\begin{array}{l}\text { Alzheimer's disease } \\
\text { control group }\end{array}$ & $57.21 \pm 3.68$ & $57.26 \pm 5.09$ & $54.70 \pm 7.57$ & $53.16 \pm 8.16$ & $53.67 \pm 8.28$ \\
\hline $\begin{array}{l}\text { Electroacupuncture } \\
\text { group }\end{array}$ & $57.65 \pm 4.15$ & $51.25 \pm 9.08$ & $49.42 \pm 14.01$ & $48.32 \pm 10.25$ & $47.10 \pm 10.78$ \\
\hline
\end{tabular}

(a)

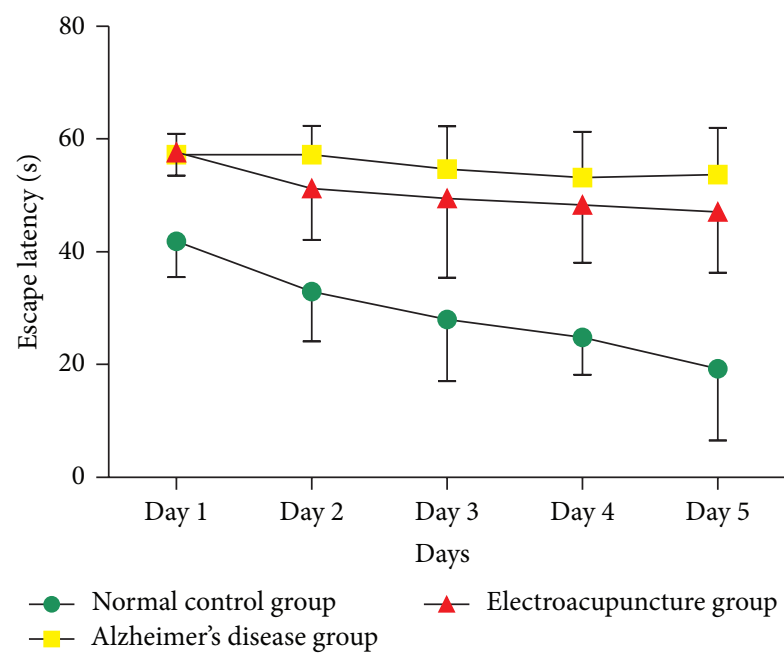

(b)

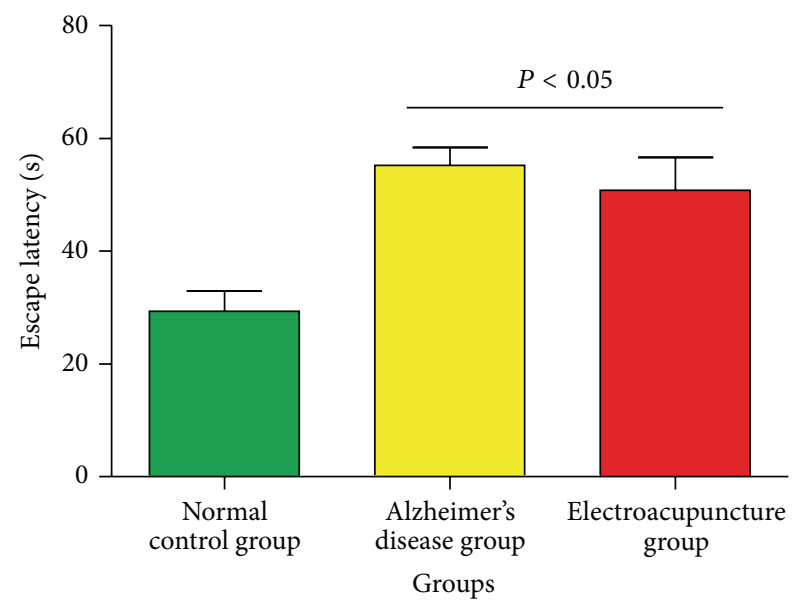

(c)

Figure 1: (a) Comparison of the escape latency of all groups. (b) The trend of the escape latency of all groups. (c) Comparison the mean of the escape latency of all groups.

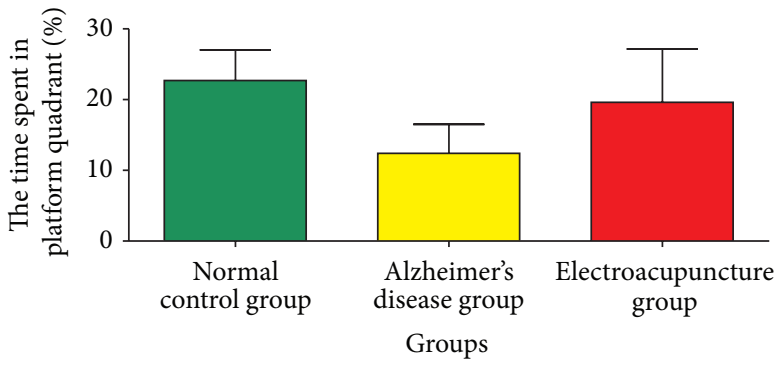

(a)

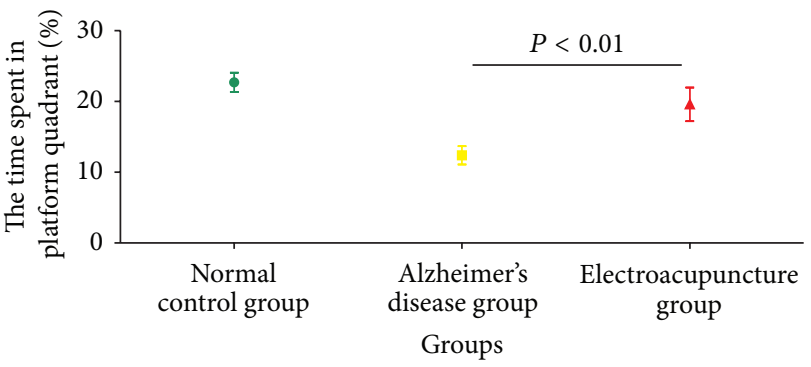

(b)

FIGURE 2: (a) The percentage of time spent in platform quadrant of each group. (b) The percentage of time spent in platform quadrant of each group.

amnesia, corpse-like syncope, aphasia from apoplexy, manicdepressive psychosis, epilepsy, hysteria, and so forth. DU 26 Shuigou is a point on the Governor Vessel, Hand-Yangming, and Foot-Yangming, also called Renzhong. Its location is on the face, at the junction of the upper $1 / 3$ and middle $1 / 3$ of the philtrum. It is used for coma, syncope, manic-depressive disorder, epilepsy, acute and chronic infantile convulsion, and so forth. EX-HN3 Yintang, an extra point, is seen in Bian Que's Jade Dragon Classics of Acupuncture and Moxibustion (Bian Que Shenying Zhenjiu Yulong Jing). It is on the forehead, at the midpoint between the eyebrows. Headache, vertigo, insomnia, and puerperal faintness are the indication of EXHN3. In general, we use the above three acupoints as the acupuncture prescription.
4.2. Senescence-Accelerated Mouse-P8 (SAMP8) Is an Optional Animal Model for Alzheimer's Disease. The senescenceaccelerated mouse (SAMP8) is a spontaneous animal model of Alzheimer's disease, and it develops early memory disturbances and changes in the blood-brain barrier resulting in decreased efflux of amyloid-beta protein from the brain [38]. This nontransgenic animal model with great utility can be better simulated for the memory deficits and the low level uptake rate of glucose in hippocampus [41]. So, in the current research, this kind of animal model can help us to find the effect of electroacupuncture treatment in curing Alzheimer's disease.

4.3. Effect of Electroacupuncture in Morris Water Maze. The Morris water maze (MWM) is one of the most common tasks 


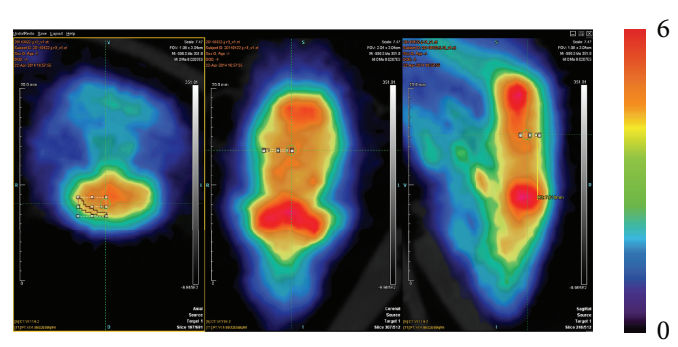

(a)

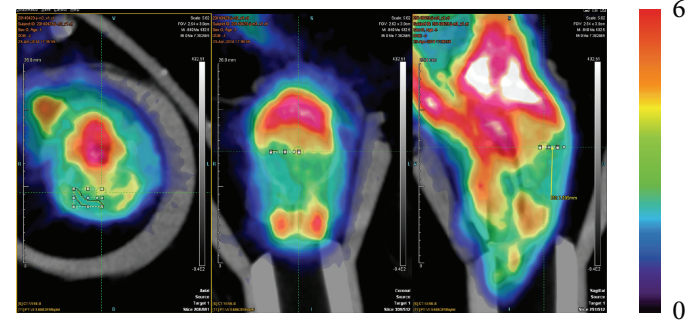

(b)

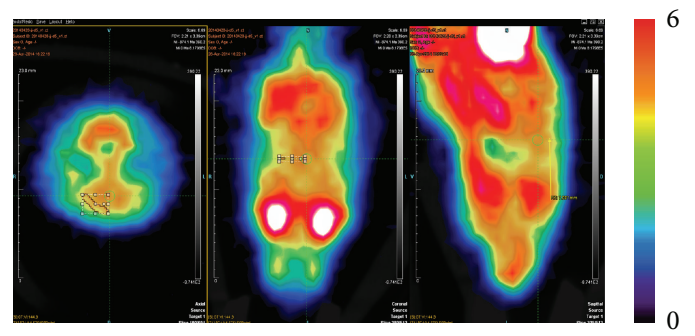

(c)

Figure 3: Right side hippocampus of the mice in micro-PET scan image. (a) Normal control group; (b) Alzheimer's disease group; (c) electroacupuncture treatment group. Color code: $\min =0, \max =6$.

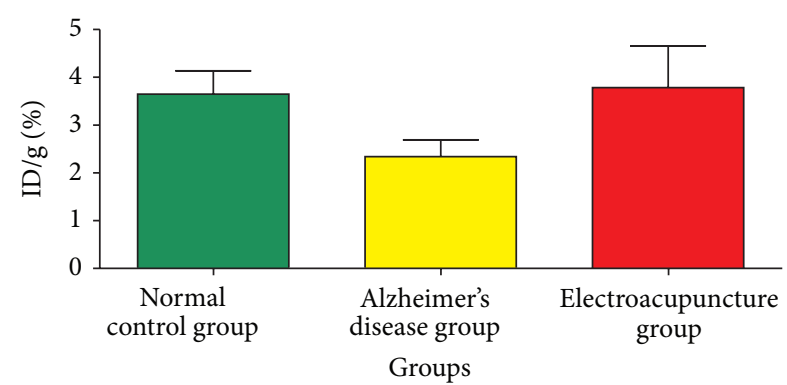

(a)

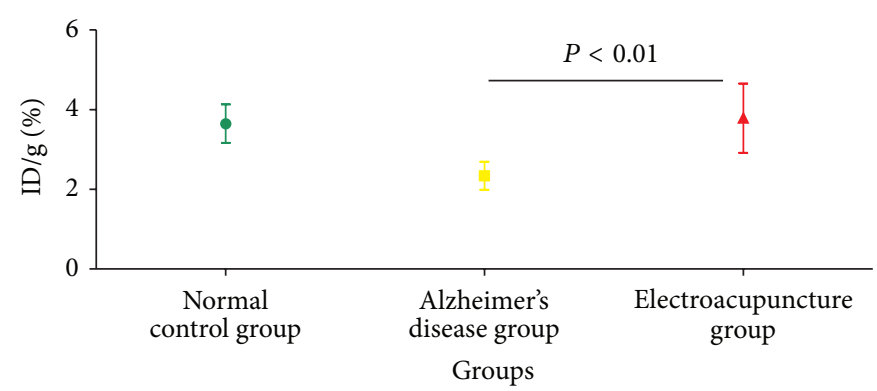

(b)

FIGURE 4: (a) The uptake rate of ${ }^{18}$ F-FDG per gram in hippocampus. (b) The uptake rate of ${ }^{18}$ F-FDG per gram in hippocampus.

used to assess spatial learning and memory ability in rodents [42]. Spatial navigation performance in the hidden goal task (HGT), a real-space human analogue of the Morris water maze, can identify mild cognitive impairment (MCI) patient with memory impairment of the hippocampus type, a known indicator of incipient Alzheimer's disease [43]. In our study, we found that after the treatment of electroacupuncture, the spatial learning and memory ability of the SAMP8 mouse had improved compared with the nontreatment SAMP8 mouse, which suggested that the electroacupuncture may improve the cognitive ability of Alzheimer's disease patients.

4.4. Effect of Electroacupuncture in Micro-PET. Studies suggested that the cognitive impairment of Alzheimer's disease to a certain extent results from the low level of uptake rate of glucose in hippocampus [44]. So in our current study, we used the micro-PET to get the in vivo image of the uptake rate of glucose in hippocampus.

Positron emission tomography is a noninvasive functional brain imaging technique at the molecular level, which makes the use of radioactive marker to analyze the metabolism condition in the brain, images the distribution of biologically targeted radiotracer with high sensitivity [45]. It can directly reflect the activity of neurons, which becomes an important tool for diagnosing disease and evaluating efficacy [46]. With the growing importance of animal research in modern molecular biology, the appearance of micropositron emission tomography (micro-PET) makes the possible of in vivo molecular imaging. Development of micro-PET instrumentation for small animal imaging and the availability of positron-emitting tracers have made this technology accessible for the noninvasive, quantitative, and repetitive imaging of biological function in living animals. The development of new probes and positron-imaging based reporter genes has extended micro-PET applications to investigations of metabolism, enzyme activity, receptor-ligand interactions, protein-protein interactions, gene expression, adoptive cell therapy, and somatic gene therapy [47].

In this research, the ${ }^{18}$ F-FDG uptake condition in the hippocampus of mice was imaged by micro-PET, which can 
show the metabolism level in the hippocampus of Alzheimer's disease mice. Seeing from the images, the ${ }^{18}$ F-FDG uptake condition of electroacupuncture treatment group is higher than Alzheimer's disease group. With further calculation and comparison of the ${ }^{18} \mathrm{~F}$-FDG uptake rate of each group, we can see that the electroacupuncture treatment group is the highest, normal control group is in the middle, and Alzheimer's disease group is the lowest. The above results showed, after the electroacupuncture treatment the glucose metabolism level in the hippocampus of the Alzheimer's disease animal model would be higher. Therefore, we could draw the conclusion that the treatment of electroacupuncture could improve the level of uptake rate of glucose in hippocampus in Alzheimer's disease animal.

\section{Conclusion}

In this research, using the test of Morris water maze and the micro-PET in Alzheimer's disease animal model SAMP8 mouse, we found that the treatment of electroacupuncture can improve the spatial learning and memory ability by heightening the level of uptake rate of glucose in hippocampus. This is an interesting notion; however, further research is needed to prove.

\section{Conflict of Interests}

The authers declare that there is no conflict of interests regarding the publication of this paper.

\section{Acknowledgments}

This research was supported by National Natural Science Foundation of China (no. 81273825).

\section{References}

[1] C. Ballard, S. Gauthier, A. Corbett, C. Brayne, D. Aarsland, and E. Jones, "Alzheimer's disease," The Lancet, vol. 377, no. 9770, pp. 1019-1031, 2011.

[2] K. Blennow, M. J. de Leon, and H. Zetterberg, “Alzheimer's disease," The Lancet, vol. 368, no. 9533, pp. 387-403, 2006.

[3] X. T. Sun, L. Jin, and P. X. Ling, "Review of drugs for Alzheimer's disease," Drug Discoveries \& Therapeutics, vol. 6, no. 6, pp. 285290, 2012.

[4] G. A. Ulett, S. Han, and J.-S. Han, "Electroacupuncture: mechanisms and clinical application," Biological Psychiatry, vol. 44, no. 2, pp. 129-138, 1998.

[5] Y. Fukazawa, T. Maeda, and S. Kishioka, "The pharmacological mechanisms of electroacupuncture," Current Opinion in Investigational Drugs (London, England: 2000), vol.10, no. 1, pp. 62-69, 2009.

[6] G. A. Ulett, "Conditioned healing with electroacupuncture," Alternative Therapies in Health and Medicine, vol. 2, no. 5, pp. 56-60, 1996.

[7] J.-W. Yang, S.-M. Jeong, K.-M. Seo, and T.-C. Nam, "Effects of corticosteroid and electroacupuncture on experimental spinal cord injury in dogs," Journal of Veterinary Science (Suwon-si, Korea), vol. 4, no. 1, pp. 97-101, 2003.
[8] H. Sumano, E. Bermudez, and K. Obregon, "Treatment of wobbler syndrome in dogs with electroacupuncture," Deutsche Tierarztliche Wochenschrift, vol. 107, no. 6, pp. 231-235, 2000.

[9] Y. Zhang, H. Xu, H. Sun, S. Chen, and F. Wang, "Electroacupuncture treatment improves neurological function associated with regulation of tight junction proteins in rats with cerebral ischemia reperfusion injury," Evidence-Based Complementary and Alternative Medicine, vol. 2014, Article ID 989340, 10 pages, 2014.

[10] C. Wang, F. Yang, X. Liu, M. Liu, Y. Zheng, and J. Guo, "Neurotrophic signaling factors in brain ischemia/reperfusion rats: differential modulation pattern between single-time and multiple electroacupuncture stimulation," Evidence-Based Complementary and Alternative Medicine, vol. 2014, Article ID 625050, 13 pages, 2014.

[11] F. Guo, W. Song, T. Jiang et al., "Electroacupuncture pretreatment inhibits NADPH oxidase-mediated oxidative stress in diabetic mice with cerebral ischemia," Brain Research, vol. 1573, pp. 84-91, 2014.

[12] F. Guo, T. Jiang, W. Song et al., "Electroacupuncture attenuates cerebral ischemia-reperfusion injury in diabetic mice through adiponectin receptor 1-mediated ohosphorylation of GSK-3 $\beta$," Molecular Neurobiology, 2014.

[13] F. Tan, J. Chen, Y. Liang et al., "Electroacupuncture attenuates cervical spinal cord injury following cerebral ischemia/reperfusion in stroke-prone renovascular hypertensive rats," Experimental and Therapeutic Medicine, vol. 7, no. 6, pp. 1529-1534, 2014.

[14] W. S. Wang, W. Z. Tu, R. D. Cheng et al., "Electroacupuncture and A-317491 depress the transmission of pain on primary afferent mediated by the $\mathrm{P} 2 \mathrm{X}_{3}$ receptor in rats with chronic neuropathic pain states," Journal of Neuroscience Research, vol. 92, no. 12, pp. 1703-1713, 2014.

[15] K. Liu, X.-Y. Gao, L. Li et al., "Neurons in the nucleus tractus solitarius mediate the acupuncture analgesia in visceral pain rats," Autonomic Neuroscience, vol. 186, pp. 91-94, 2014.

[16] H. Li, S. Hu, J. Zhang et al., "Effects and mechanisms of auricular electroacupuncture on visceral pain induced by colorectal distension in conscious rats," Acupuncture in Medicine, vol. 32, no. 6, pp. 472-477, 2014.

[17] Y.-H. Gao, J.-Y. Wang, L.-N. Qiao et al., "NK cells mediate the cumulative analgesic effect of electroacupuncture in a rat model of neuropathic pain," BMC Complementary and Alternative Medicine, vol. 14, no. 1, p. 316, 2014.

[18] X.- M. Chen, J. Xu, J.-G. Song, B.-J. Zheng, and X.R. Wang, "Electroacupuncture inhibits excessive interferongamma evoked up-regulation of $\mathrm{P} 2 \mathrm{X} 4$ receptor in spinal microglia in a CCI rat model for neuropathic pain," British Journal of Anaesthesia, vol. 114, no. 1, pp. 150-157, 2014.

[19] W.-T. Chen, F.-C. Chang, Y.-H. Chen, and J.-G. Lin, "An evaluation of electroacupuncture at the Weizhong acupoint (BL-40) as a means of relieving pain induced by extracorporeal shock wave lithotripsy," Evidence-Based Complementary and Alternative Medicine, vol. 2014, Article ID 592319, 8 pages, 2014.

[20] Z. Y. Ju, H. S. Cui, X. H. Guo, H. Y. Yang, J. S. He, and K. Wang, "Molecular mechanisms underlying the effects of acupuncture on neuropathic pain," Neural Regeneration Research, vol. 8, no. 25, pp. 2350-2359, 2013.

[21] Z. J. Zhang, R. Ng, S. C. Man et al., "Use of electroacupuncture to accelerate the antidepressant action of selective serotonin reuptake inhibitors: a single-blind, randomised, controlled 
study, Hong Kong Medical Journal, vol. 19, supplement 9, pp. 12-16, 2013.

[22] D. K. Weiner, C. G. Moore, N. E. Morone, E. S. Lee, and C. Kent Kwoh, "Efficacy of periosteal stimulation for chronic pain associated with advanced knee osteoarthritis: a randomized, controlled clinical trial," Clinical Therapeutics, vol. 35, no. 11, pp. 1703.e5-1720.e5, 2013.

[23] M. L. Yeh, Y. C. Chung, K. M. Chen, M. Y. Tsou, and H. H. Chen, "Acupoint electrical stimulation reduces acute postoperative pain in surgical patients with patient-controlled analgesia: a randomized controlled study," Alternative Therapies in Health and Medicine, vol. 16, no. 6, pp. 10-18, 2010.

[24] Y. X. Chen, K. M. Kong, W. D. Wang, C. H. Xie, and R. H. $\mathrm{Wu}$, "Functional MR imaging of the spinal cord in cervical spinal cord injury patients by acupuncture at LI 4 (Hegu) and LI 11(Quchi)," in Proceedings of the 29th Annual International Conference of IEEE-EMBS, Engineering in Medicine and Biology Society (EMBC '07), pp. 3388-3391, August 2007.

[25] A. M. K. Wong, C.-P. Leong, T.-Y. Su, S.-W. Yu, W.-C. Tsai, and C. P. C. Chen, "Clinical trial of acupuncture for patients with spinal cord injuries," The American Journal of Physical Medicine \& Rehabilitation, vol. 82, no. 1, pp. 21-27, 2003.

[26] H. Zhang, T. Kang, L. Li, and J. Zhang, "Electroacupuncture reduces hemiplegia following acute middle cerebral artery infarction with alteration of serum NSE, S-100B and endothelin," Current Neurovascular Research, vol. 10, no. 3, pp. 216-221, 2013.

[27] J. Sun, H. Sang, and C. Yang, "Electroacupuncture improves orthostatic tolerance in healthy individuals via improving cardiac function and activating the sympathetic system," Europace, vol. 15, no. 1, pp. 127-134, 2013.

[28] K.-W. Yu, C.-L. Lin, C.-C. Hung et al., "Effects of electroacupuncture on recent stroke inpatients with incomplete bladder emptying: a preliminary study," Clinical Interventions in Aging, vol. 7, pp. 469-474, 2012.

[29] W. T. Hsing, M. Imamura, K. Weaver, F. Fregni, and R. S. Azevedo Neto, "Clinical effects of scalp electrical acupuncture in stroke: a sham-controlled randomized clinical trial," The Journal of Alternative and Complementary Medicine, vol. 18, no. 4, pp. 341-346, 2012.

[30] S. P. Zhang, T. T. Chiu, and S. N. Chiu, "Long-term efficacy of electroacupuncture for chronic neck pain: a randomised controlled trial," Hong Kong Medical Journal, vol. 19, supplement 9, pp. 36-39, 2013.

[31] B.-J. Wan, W. Huang, Y.-X. Zhang, and H.-S. Zhang, "Influence of electroacupuncture with penetration needling method on comprehensive pain score in patients with cervical spondylotic radiculopathy," Chinese Acupuncture \& Moxibustion, vol. 33, no. 5, pp. 407-410, 2013.

[32] Z.-R. Sun, J.-H. Yue, and Q.-H. Zhang, "Electroacupuncture at Jing-jiaji points for neck pain caused by cervical spondylosis: a study protocol for a randomized controlled pilot trial," Trials, vol. 14, no. 1, article 360, 2013.

[33] S. Mucuk, M. Baser, and T. Ozkan, "Effects of noninvasive electroacupuncture on labor pain, adrenocorticotropic hormone, and cortisol," Alternative Therapies in Health and Medicine, vol. 19, no. 3, pp. 26-30, 2013.

[34] S. Lee, J.-H. Kim, K.-M. Shin et al., "Electroacupuncture to treat painful diabetic neuropathy: study protocol for a three-armed, randomized, controlled pilot trial," Trials, vol. 14, article 225, 2013.
[35] C. C. L. Xue, R. D. Helme, S. Gibson et al., "Effect of electroacupuncture on opioid consumption in patients with chronic musculoskeletal pain: protocol of a randomised controlled trial," Trials, vol. 13, article 169, 2012.

[36] K.-F. Chung, W.-F. Yeung, Z.-J. Zhang et al., "Randomized noninvasive sham-controlled pilot trial of electroacupuncture for postpartum depression," Journal of Affective Disorders, vol. 142, no. 1-3, pp. 115-121, 2012.

[37] Y. O. Cakmak, "A review of the potential effect of electroacupuncture and moxibustion on cell repair and survival: the role of heat shock proteins," Acupuncture in Medicine, vol. 27, no. 4, pp. 183-186, 2009.

[38] J. E. Morley, H. J. Armbrecht, S. A. Farr, and V. B. Kumar, “The senescence accelerated mouse (SAMP8) as a model for oxidative stress and Alzheimer's disease," Biochimica et Biophysica Acta, vol. 1822, no. 5, pp. 650-656, 2012.

[39] K. Bromley-Brits, Y. Deng, and W. Song, "Morris water maze test for learning and memory deficits in Alzheimer's disease model mice," Journal of Visualized Experiments, no. 53, article 2920, 2011.

[40] Z. B. Wang Yongyan, Encephalopathy of Chinese Medicine, People's Health Publishing House, Beijing, China, 2007.

[41] D. S. Woodruff-Pak, "Animal models of Alzheimer's disease: therapeutic implications," Journal of Alzheimer's Disease, vol. 15, no. 4, pp. 507-521, 2008.

[42] J. F. Ge, C. C. Qi, J. P. Qiao, C. W. Wang, and J. N. Zhou, "Sex differences in ICR mice in the morris water maze task," Physiological Research, vol. 62, no. 1, pp. 107-117, 2013.

[43] J. Laczó, R. Andel, M. Vyhnalek et al., "From morris water maze to computer tests in the prediction of Alzheimer's disease," Neurodegenerative Diseases, vol. 10, no. 1-4, pp. 153-157, 2012.

[44] L. Mosconi, R. Mistur, R. Switalski et al., "FDG-PET changes in brain glucose metabolism from normal cognition to pathologically verified Alzheimer's disease," European Journal of Nuclear Medicine and Molecular Imaging, vol. 36, no. 5, pp. 811-822, 2009.

[45] B. Foster, U. Bagci, A. Mansoor, Z. Xu, and D. J. Mollura, "A review on segmentation of positron emission tomography images," Computers in Biology and Medicine, vol. 50, pp. 76-96, 2014.

[46] S. E. Schindler, J. McConathy, B. M. Ances, and M. I. Diamond, "Advances in diagnostic testing for Alzheimer disease," Missouri Medicine, vol. 110, no. 5, pp. 401-405, 2013.

[47] H. R. Herschman, "Micro-PET imaging and small animal models of disease," Current Opinion in Immunology, vol. 15, no. 4, pp. 378-384, 2003. 


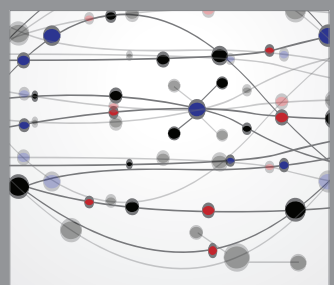

The Scientific World Journal
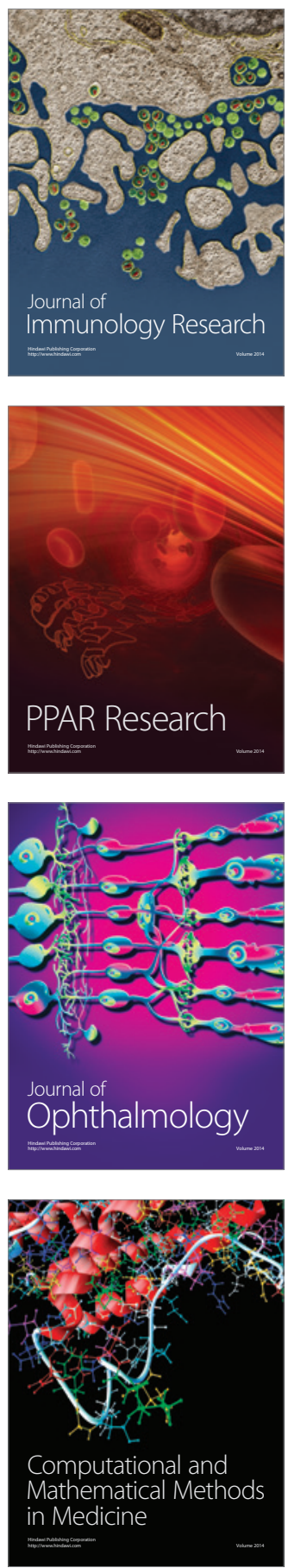

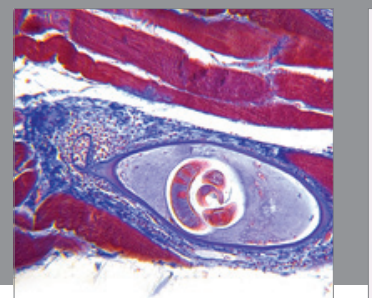

Gastroenterology

Research and Practice
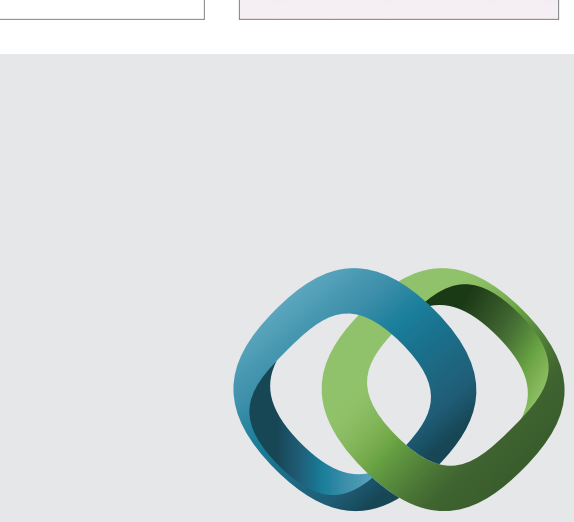

\section{Hindawi}

Submit your manuscripts at

http://www.hindawi.com
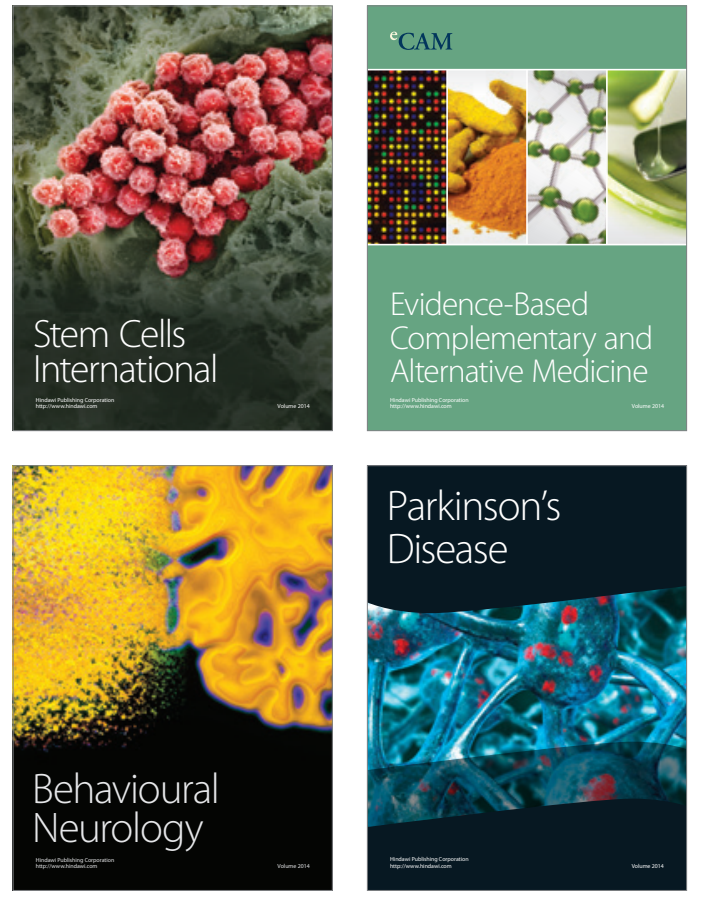
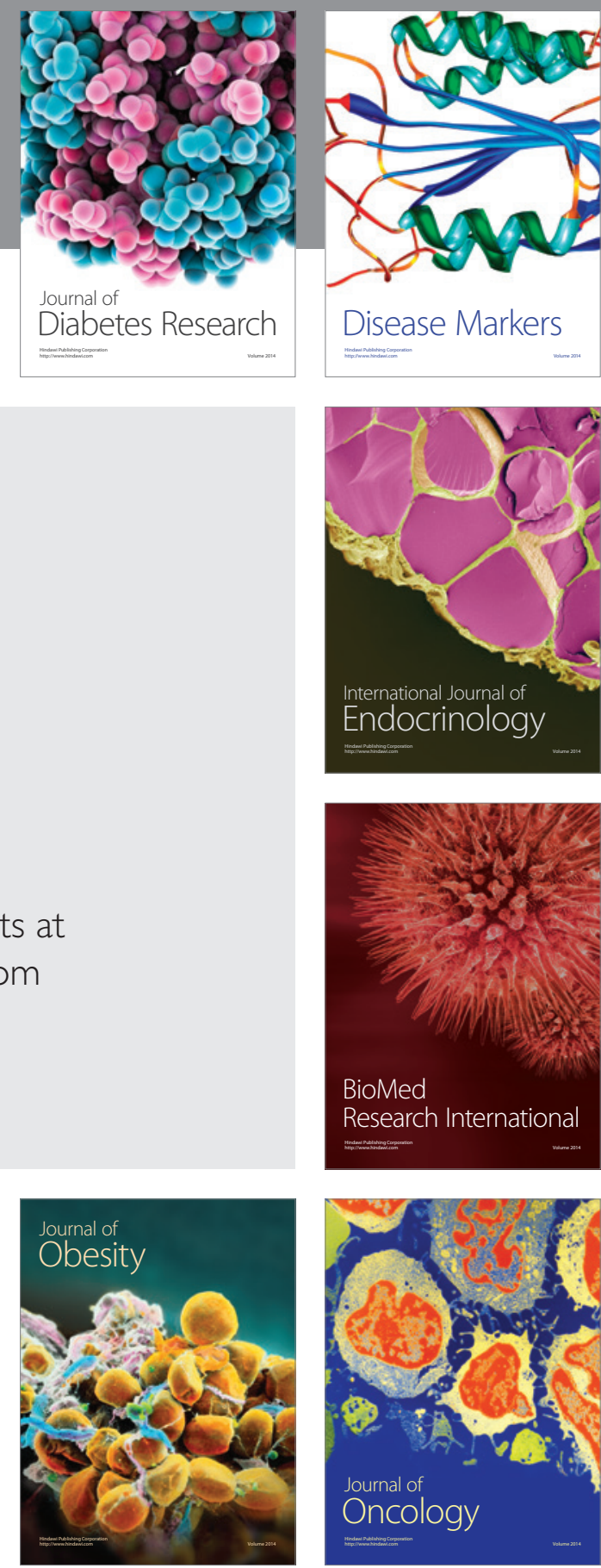

Disease Markers
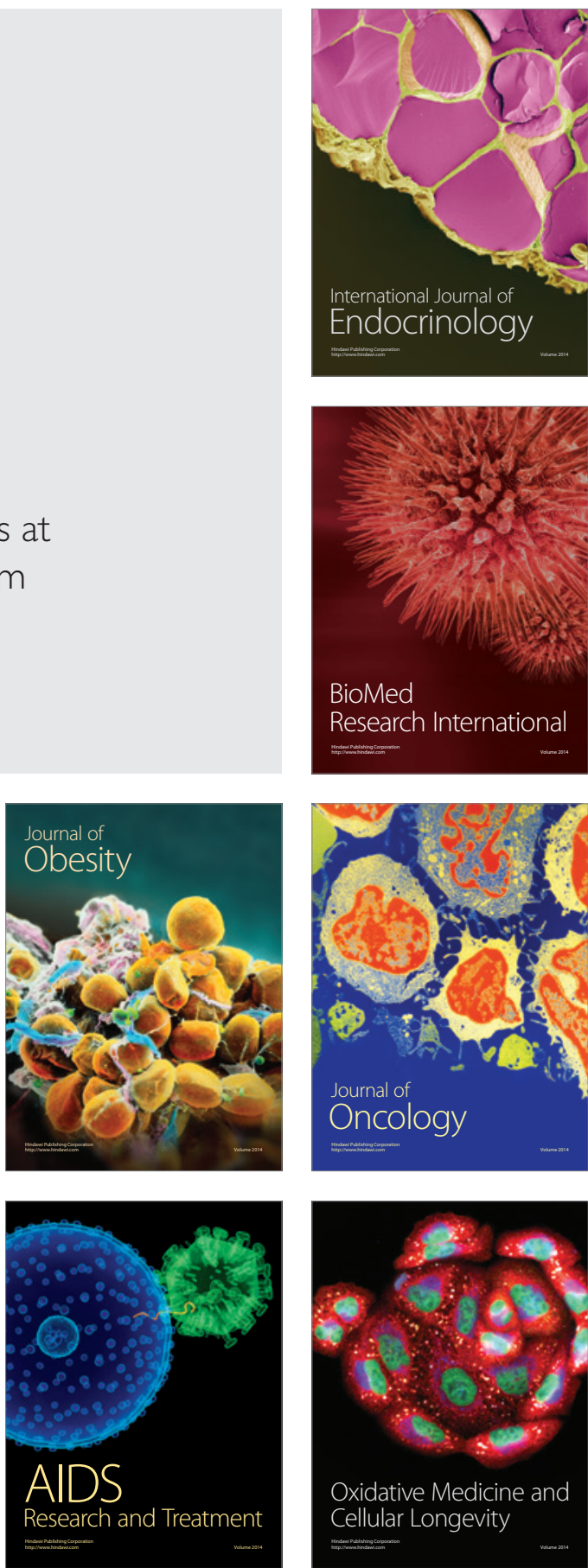